\title{
Comparison Study of Time Synchronization in NC-OFDM Systems Based on Symmetric Correlator
}

\author{
Tajul Miftahushudur, Suyoto* \\ Pusat Penelitian Elektronika dan Telekomunikasi \\ Lembaga Ilmu Pengetahuan Indonesia \\ Komplek LIPI Gd 20, Jl Sangkuriang 21/54D \\ Bandung, Indonesia
}

\begin{abstract}
NC-OFDM as one of the candidates for the Cognitive Radio (CR) system has many challenges in the synchronizing time. This is due to the frequency sharing which can cause Narrowband Interference (NBI), which causes a very significant time synchronization error. Large errors in time synchronization can reduce overall NCOFDM system performance. In this study, we evaluate the time synchronization performance of NC-OFDM systems on the multipath channel in the present of NBI. We provide an evaluation of the time synchronization performance by comparing the time synchronization algorithm that has a timing metric such as an impulse-like (Symmetric correlator). Here, we present three algorithms that use the symbol training to calculate the arrival time of the NC-OFDM symbol. The simulation conducted by considering the effects of multipath channel, Signal to Noise Ratio (SNR), Carrier Frequency Offset (CFO), and SIR (Signal to Interference Ratio). SIR is modeled with NBI. Algorithm performance is measured by MAE (Mean Absolute Error) and MSE (Mean Squared Error). Simulation results show that the autocorrelation-based method has very poor performance in MAE and MSE tests, so it is not suitable for NC-OFDMbased Cognitive Radio systems. The GLRT (Generalized Likelihood Ratio Test) method and Iterative Normalization method perform well in case the NC-OFDM Cognitive Radio systems.
\end{abstract}

Keywords: NC-OFDM, time synchronization, CR, NBI.

\section{INTRODUCTION}

Cognitive radio has received much attention from researchers to meet the increasing need for spectrum sources [1] and [2]. One technique on the physical layer that can be used to exploit an empty spectrum in an unused band is NC-OFDM (Non-Continuous OFDM (Orthogonal Frequency Division Multiplexing)). This is done by adjusting the parameters of the wireless communication system to automatically follow the communication environment, for example, LU (license users) share frequency bands with CR users. However, the presence of an NBI can disrupt the time synchronization performance, where the NBI signal can be detected as a synchronization signal. Therefore, some researchers conduct evaluations and provide solutions in overcoming the problem of time synchronization.

The most popular technique for time synchronization is the technique proposed by Schmidl and Cox $(\mathrm{S} \& \mathrm{C})$ [3]. This technique uses the correlation of two identical parts in the time domain for time synchronization, where two identical parts are generated with a series of PNs (Pseudo Noise) in the frequency domain on even subcarriers or on odd subcarriers. In [3] evaluating the performance of $\mathrm{S} \& \mathrm{C}$ techniques on $\mathrm{CR}$ systems, where the interference being modeled is white

\footnotetext{
* Corresponding Author.

Email: suyo003@lipi.go.id

Received: September 30, 2019 ; Revised: November 25, 2019

Accepted: November 29, 2019 ; Published: December 31, 2019

(C) 2019 PPET - LIPI
}

noise and NBI. As shown in [4], the S\&C technique experienced a time synchronization error due to an NBI signal being detected as a synchronization signal. In [5] it is also shown that the time synchronization error due to NBI occurs when the SIR is smaller than $15 \mathrm{~dB}$.

The weakness of the S\&C technique for time synchronization in OFDM / NC-OFDM systems is that there is a flat area on the timing metric, resulting in a wide estimation variant. There are other techniques that produce sharper timing metrics, including the techniques proposed by Park et al. [6] (Park technique) and Yi et al. [7] (YI technique) which uses symmetric correlators. The YI technique is generally better than the Park technique, this is because the timing matrices of the YI technique have sidelobes much smaller than the park technique. Higher sidelobes can decrease the performance of timing synchronization in a high noise environment. In [8] improvements were made to the synchronization technique based on symmetric correlators in the face of high delay spread in OFDM systems with the GLRT (Generalized Likelihood Ratio Test) technique. In [9] improvements were made to the GLRT technique in order to improve detection of the arrival of the OFDM symbol in multipath channel environments with very high delay spreads in OFDM systems with iterative normalization technique.

Therefore, this research will evaluate the YI technique [7] along with its improvement techniques for symmetric correlators [8]-[9] in case OFDM-based CR systems (NC-OFDM), where the disturbance arises is the 
NBI. The rest of this paper is organized as follows. Section II describes the NC-OFDM system model. Section III overviews the time synchronization scheme based on symmetric correlation. Section IV describes the simulation result in and section $\mathrm{V}$ gives the conclusion.

\section{NC-OFDM SYSTEM MODEL}

Figure 1 illustrates the similarity of NC-OFDM based discrete complex baseband CR system, the cognitive user sends $\boldsymbol{x}$ vector symbols using $N$ subcarriers, where the subcarriers that can be used are adapted to the sensing system. The data is modulated after IFFT is converted from parallel to serial and added with a cyclic prefix of length $L_{C P}$. Thus, the OFDM baseband signal can be written as in (1):

$$
d^{k}=F_{d} x^{k}
$$

where $k$ denotes the sequence of symbols, $F_{d}(N+$ $\left.L_{C P}\right) \times N$ is a matrix, $N$ is the number of subcarriers and each element in the matrix $F_{d}$ is defined as $F_{d}(n, m)=$ $\frac{1}{\sqrt{N}} \exp \left(\frac{j 2 \pi n m}{N}\right),-L_{C P} \leq n<N, 0 \leq m<N$, where $n$ and $m$ respectively represent the interval normalization of sampling time intervals. NC-OFDM signals sent in frame form can be written as in (2):

$$
d_{f}(n)=\sum_{k=0}^{K-1} d^{k}
$$

At the receiver, the received signal is distorted due to multipath channel, NBI, white noise, and CFO in (3):

$$
r(n)=b(n) e^{j 2 \pi v n / N}+\sqrt{\sigma_{i}^{2}} e^{\frac{j 2 \pi f_{N B I} n}{N}+j \varphi}+z(n),
$$

where:

$$
b(n)=\sum_{l=0}^{L_{C H}-1} d_{f}(n-l) h(l),
$$

$b(n)$ is the NC-OFDM signal at the receiver disturbed by multipath channel, $h(l)$ is the channel coefficient at the $l$-th path, $l$ is the index of the channel path, $L_{C H}$ is the channel length, and $v$ is the normalization of the $\mathrm{CFO}$ with respect to the subcarrier spacing. In (3), the second part $\sigma_{i}^{2}$ is the power modeling of the NBI, $f_{N B I}$ is the normalization of the NBI frequency, and $\varphi$ is the phase of the NBI signal. The third part of (3) is white Gaussian noise with mean zero and variance $\sigma_{z}^{2}$.

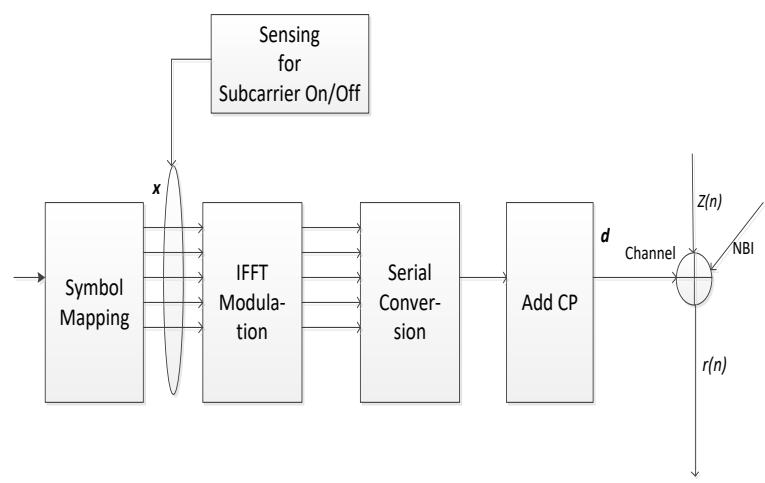

Figure 1. NC-OFDM with subcarrier on/off.

\section{TIME SYNCHRONIZATION SCHEME BASED ON SYMMETRIC CORRELATION}

In this section, a brief review of time synchronization based on symmetrical correlations and their improvement techniques are given.

\section{A. The YI Method}

The form of training symbol in the frequency domain in [7] is designed as in (5).

$$
\begin{gathered}
X_{\text {preamble }}=[\underbrace{0, \ldots 0}_{n_{f}}, X_{1}, X_{2}, \ldots, X_{D}, \underbrace{0, \ldots, 0}_{n_{k}}] \\
N=n_{f}+D+n_{k}
\end{gathered}
$$

and in the time domain, the training symbol can be represented as in (6).

$$
x_{\text {preamble }}=\left[s_{0}, s_{1}, \ldots, s_{N-1}\right]
$$

where $D$ is the number of active subcarriers, $X_{i}, i=$ $1,2, \ldots, D$ is the real value of the PN series (Pseudo Noise), a series with length $n_{f}+n_{k}$ is zero which is used as a guard band. Because the values of $X_{i}, i=1,2, \ldots, D$ are real, then $s_{N-n}=s_{n}^{*}, n=1, \ldots, \frac{N}{2}-1$.

The form of training symbol for symmetric correlator in the time domain can be described as in Figure 2. Where F represents a sample of length $N / 2$, and $\mathbf{G}$ is the symmetric conjugate of $\mathbf{F}$. So that the symmetric correlator that utilizes the properties of $\mathbf{G}$ which is a symmetric conjugate of $\mathbf{F}$ can be expressed with the timing metric as in (7).

$$
M(n)=\frac{|p(n)|^{2}}{(R(n))^{2}},
$$

where:

$$
\begin{gathered}
p(n)=\sum_{q=1}^{\frac{N}{2}-1} r(n+q) \cdot r(n-q+N), \\
R(n)=\sum_{q=1}^{\frac{N}{2}-1}|r(n+k)|^{2} .
\end{gathered}
$$

Furthermore, the calculation of time offset is done as in (10).

$$
\hat{\epsilon}_{Y I}=\underbrace{\operatorname{argmax}}_{n}(M(n)) .
$$

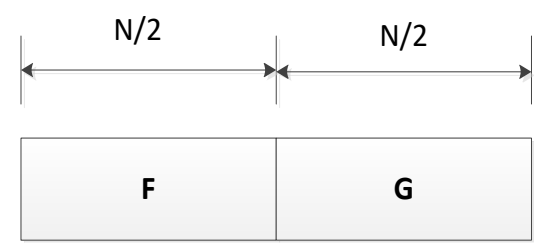

Figure 2. Time domain representation of training symbol for the symmetric correlator. 


\section{B. The GLRT Method}

The GLRT technique utilizes the property of symmetric correlation in (8) by taking the absolute value which can be stated as in (11).

$$
t(n)=|p(n)|
$$

To derive the PDF (probability distribution function) from $t(n)$, first define $\rho t(n)$ as in (12).

$$
\rho t(n)=\sum_{q=1}^{\frac{N}{2}-1} r(n+q) \cdot r(n-q+N)
$$

for $\left|n-n_{0}\right|<L_{F}, \rho t(n)$ follows the normal complex distribution as in (13).

$$
p t(n) \sim \begin{cases}C N\left(0, T \sigma_{r}^{4}\right) & n \notin L, \\ C N\left(T h^{2}\left(n-n_{0}\right) \sigma_{x}^{2} e^{2 \pi v}, T \sigma_{r}^{4}\right) & n \in L,\end{cases}
$$

where $L_{F}$ is the number of identical parts in the training symbol $L_{F}=N / 2, T=\frac{N}{2}-1, \sigma_{r}^{2}=\varkappa \sigma_{x}^{2}+\sigma_{z}^{2}, \varkappa=$ $\sum_{l}|h(l)|^{2}, \sigma_{x}^{2}=\frac{1}{N} \sum_{q=0}^{N-1}\left|x_{p}(q)\right|^{2}, \sigma_{z}^{2}$ is the noise variant, and $x_{p}(q)$ is the training signal in the time domain. $n_{0}$ Indicates the start of the training symbol at $n=n_{0}$, which relates to the arrival of the first path of NC-OFDM symbols and $L$ is the multipath channel index $L=$ $n_{0}, n_{0}+1, \ldots, n_{0}+L_{C H}$. Therefore, for the symmetric correlator with a length $\left|n-n_{0}\right|<L_{F}, t(n)$ is a Rician random variable with PDF as in (14).

$f\left(t(n) ; \sigma^{2}, \theta(n)\right)$

$=\frac{t(n)}{\sigma^{2}} \exp \left(-\frac{t(n)+\theta(n)}{2 \sigma^{2}}\right) I_{0}\left(\frac{t(n) \theta(n)}{\sigma^{2}}\right)$,

where $I_{0}$ is the first type of Bassel function of order 0 ,

$$
\theta(n)= \begin{cases}T\left|h^{2}\left(n-n_{0}\right)\right| \sigma_{x}^{2}, & n \in L \\ 0, & n \notin L,\end{cases}
$$

and $\sigma^{2}=T \sigma_{r}^{4} / 2$

From the PDF that was revealed in (14), Cho and Park investigate the statistical change from $t(n)$ when the training symbol is received. Then with the GLRT approach, the timing metric is defined as in (16).

$$
\begin{aligned}
& M_{G L R T}(n) \\
& =\exp \left(-\frac{1}{2} \Phi(n) 1\right) I_{0}\left(\sqrt{\Phi^{2}(n)-2 \Phi(n)}\right),
\end{aligned}
$$

with:

$$
\begin{aligned}
\Phi(n) & =\frac{t^{2}(n)}{\sigma_{0}^{2}(n)}, \\
\sigma_{0}^{2}(n) & =\frac{1}{2 J} \sum_{j=0}^{J-1} t^{2}(n-j) .
\end{aligned}
$$

The parameter $J$ ( $\geq$ channel length) in (18) is the length of samples for observation to detect the first arriving path of the NC-OFDM symbol. Thus, the calculation of the arrival time (time offset) of the NC-OFDM symbol can be defined as in (19).

$$
\hat{\epsilon}_{G L R T}=\underbrace{\operatorname{argmax}}_{n}\left(M_{G L R T}^{\prime}(n)\right),
$$

with:

$$
M_{G L R T}^{\prime}(n)=\left\{\begin{array}{c}
M_{G L R T}(n), \quad t(n)>\lambda, \\
0, \text { others, }
\end{array}\right.
$$

and $\lambda$ is the threshold, which is set to avoid False Alarm (FA) in (21). Probability of FA $\left(P_{F A}\right)$ derived from (14) when $\theta(n)=0$ as in (21).

$$
P_{F A}=\exp \left(-\frac{\lambda}{2 \sigma^{2}}\right)
$$

if $\sigma^{2}$ is replaced with $\sigma_{0}^{2}$, the threshold can be determined for the False Alarm rate that has been given as in (22).

$$
\lambda=\sqrt{-2 \sigma_{0}^{2} \log P_{F A}}
$$

\section{The Iterative Normalization Method}

The Iterative Normalization technique as in [9] can be written as in (23).

$$
Z_{i}(n)=\sqrt{\frac{Z_{i-1}^{2}(n)}{Z_{Z(i-1)}^{2}(n)}}
$$

where $i$ is the index of iteration and $\sigma_{Z i}^{2}(n)$ is a variance of the symmetric correlator on the $i$ iteration which is defined as in (24).

$$
\sigma_{Z i}^{2}(n)=\frac{1}{N_{\text {norm }}} \sum_{k=0}^{N_{\text {norm }}-1} Z_{i}^{2}(n-k),
$$

with $N_{\text {norm }}$ is the length of observation for iterative normalization.

The Iterative Normalization Method is carried out as follows. First, determine the value of $Z_{0}(n)=t(n)$, and then the iteration process is carried out as in (23) for $i=$ 1 to $\Gamma$, where $\Gamma$ is the number of iterations. After obtaining the value of $Z_{\Gamma}(n)$, redefine the value of $t(n)=Z_{\Gamma}(n)$. Then the timing estimation of the arrival of the OFDM symbol is done by the GRLT method, so that the Iterative Normalization method can be written as in (25).

$$
\begin{aligned}
M_{N I}(n)=\exp ( & -\frac{1}{2} \Phi(n) \\
& +1) I_{0}\left(\sqrt{\Phi^{2}(n)-2 \Phi(n)}\right),
\end{aligned}
$$

where $\Phi(n)$ is defined in (17) and $\sigma_{0}^{2}$ are defined in (18). 


\section{Simulation RESUltS}

In this section, we evaluate the performance of three algorithms in terms of timing metrics and measure MAE and MSE from the timing point. For the parameters of NC-OFDM simulations can be seen in Table 1 . Simulation is done under the vehicular $\mathbf{B}$ [10] multipath channel model. The parameters of the vehicular $\mathbf{B}$ channel for the simulation are given in Table 2. In this simulation, the NC-OFDM frame is sent preceded by 2 empty symbols.

\section{A. Timing Metric Evaluation}

First, we evaluate the timing metric of three algorithms based on symmetric correlation in conditions without NBI (SIR $=\sim d B)$. Then we evaluated the three algorithms under conditions of NBI (SIR $=0 \mathrm{~dB}$ ). Figure 3 shows the results of the timing metric simulation of the three algorithms, it can be seen that the YI algorithm has decreased performance in channel conditions with a fairly high delay spread. This happens because the YI algorithm has delayed calculations due to the high delay spread. GLRT algorithm and Iterative Normalization algorithm can work well in channel conditions with high delay spread.

In Figure 4, it can be seen that the YI algorithm has a quite large synchronization error, this is due to the presence of the NBI, so that the NBI signal is detected as a synchronization signal. The improvement from the YI method, namely the GLRT technique and the Iterative Normalization technique can overcome NBI well, this is due to the two techniques using statistical techniques that compare the distribution of training signal with signals without training.
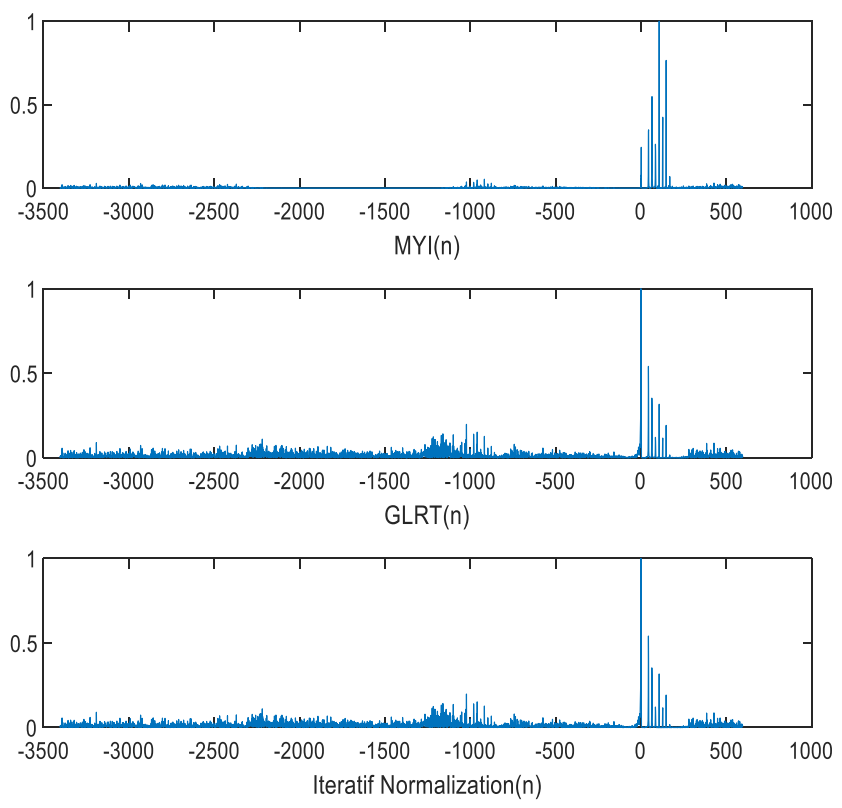

Figure 3. Timing metric comparison without NBI $(\mathrm{SIR}=\sim \mathrm{dB})$ for $\mathrm{SNR}=20 \mathrm{~dB}$
TABLE 1

SIMULATION PARAMETERS FOR NC-OFDM SYSTEMS

\begin{tabular}{|c|c|c|}
\hline No. & Parameter & Value \\
\hline 1. & FFT size & 2048 \\
\hline 2. & $\begin{array}{c}\text { Data modulation } \\
\text { technique }\end{array}$ & 16 -QAM \\
\hline 3. & CP length & $\begin{array}{c}12.5 \% \text { of the NC-OFDM } \\
\text { symbol }\end{array}$ \\
\hline 4. & Sampling rate & $0.1 \mu s$ \\
\hline 5. & Channel model & vehicular $\mathbf{B}$ \\
\hline 6. & $v$ & 0.9 \\
\hline 7. & Vehicle speed & $120 \mathrm{~km} / \mathrm{hour}$ \\
\hline 8. & $P_{F A}$ & 100 \\
\hline 9. & $\varphi$ & $1024-1073(50$ subcarriers $)$ \\
\hline 10. & LU subcarriers & $L_{C P} / 2$ \\
\hline 9. & $J$ & $N / 2$ \\
\hline 10. & $N_{\text {norm }}$ & \\
\hline
\end{tabular}

TABLE 2

SIMULATION PARAMETERS FOR VEHICULAR B MULTIPATH CHANNEL MODEL

\begin{tabular}{|c|c|c|}
\hline $\begin{array}{c}\text { Number of } \\
\text { Channel Path }\end{array}$ & Delay $(\boldsymbol{\mu s})$ & Power (dB) \\
\hline \multirow{2}{*}{6} & 0.0 & -2.5 \\
\cline { 2 - 3 } & 0.3 & 0 \\
\cline { 2 - 3 } & 8.9 & -12.8 \\
\cline { 2 - 3 } & 12.9 & -10 \\
\cline { 2 - 3 } & 17.1 & -25 \\
\cline { 2 - 3 } & 20.0 & -16 \\
\hline
\end{tabular}
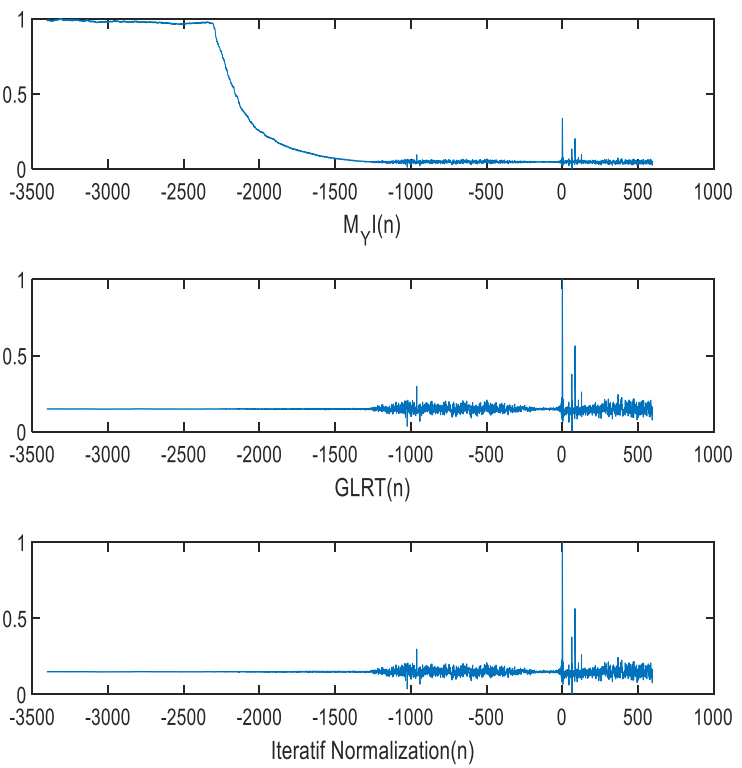

Figure 4. Timing metric comparison in the present of NBI $(\mathrm{SIR}=0$ dB) for $S N R=20 \mathrm{~dB}$.

\section{B. MAE Evaluation}

Figures 5, 6, 7, 8 and 9 show the results of MAE simulation for $\mathrm{SIR}=0 \mathrm{~dB}, \mathrm{SIR}=5 \mathrm{~dB}, \mathrm{SIR}=10 \mathrm{~dB}, \mathrm{SIR}$ $=15 \mathrm{~dB}$, and SIR $=20 \mathrm{~dB}$. It can be seen that the YI algorithm experiences a considerable time synchronization error, this is due to the autocorrelation technique based on symmetric correlator, the NBI signal is detected as a time synchronization signal. The GLRT algorithm and Iterative Normalization algorithm can 
work quite well in the presence of NBI. This is because both algorithms use autocorrelation techniques which are improved by normalization techniques. This normalization technique compares the output distribution of symmetric correlator signals.

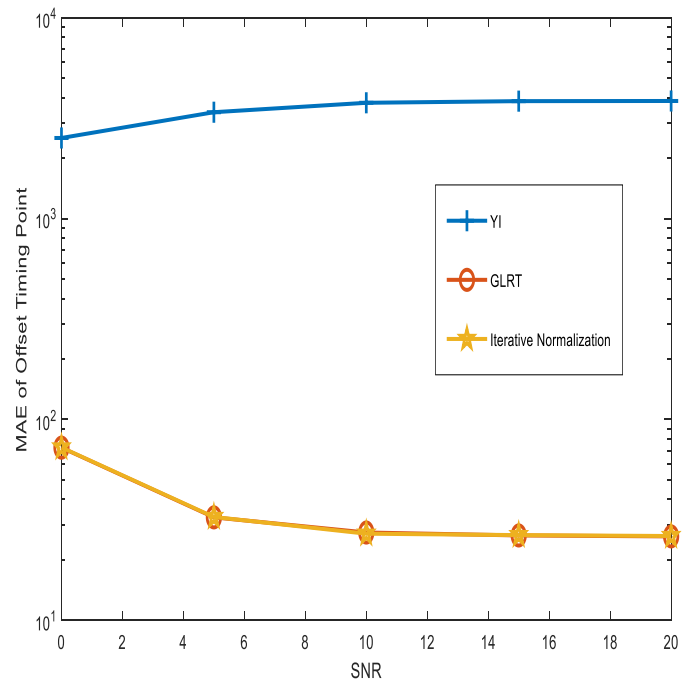

Figure 5. Simulation results for timing estimation measure in MAE for $\operatorname{SIR}=0 \mathrm{~dB}$.

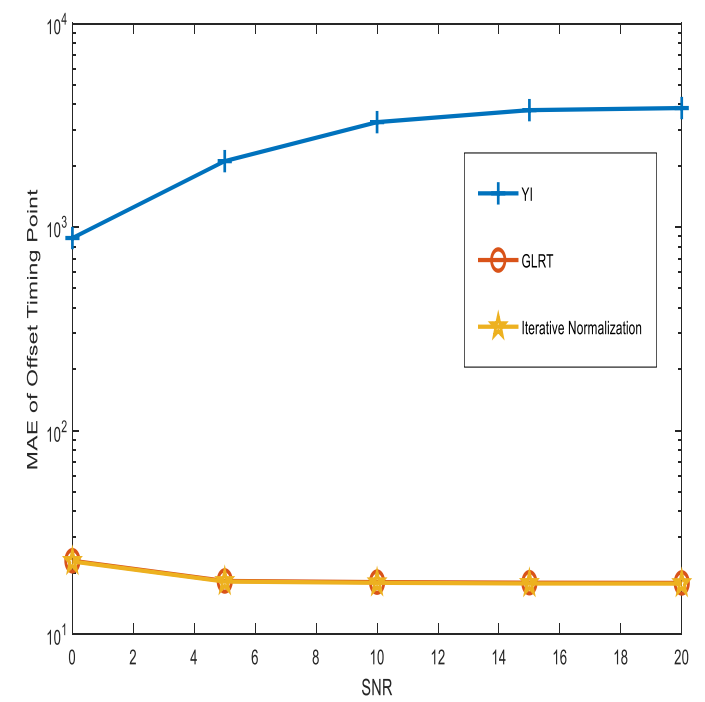

Figure 6. Simulation results for timing estimation measure in MAE for $\operatorname{SIR}=5 \mathrm{~dB}$.

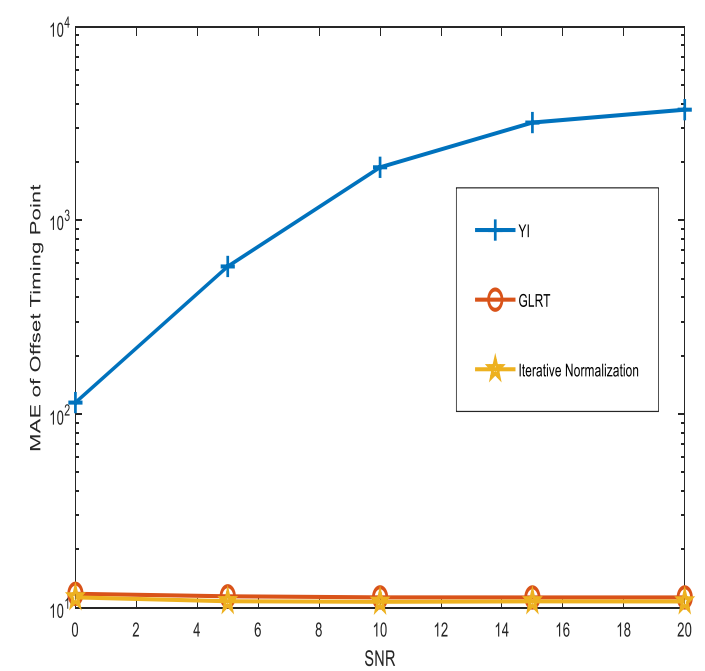

Figure 7. Simulation results for timing estimation measure in MAE for $\mathrm{SIR}=10 \mathrm{~dB}$

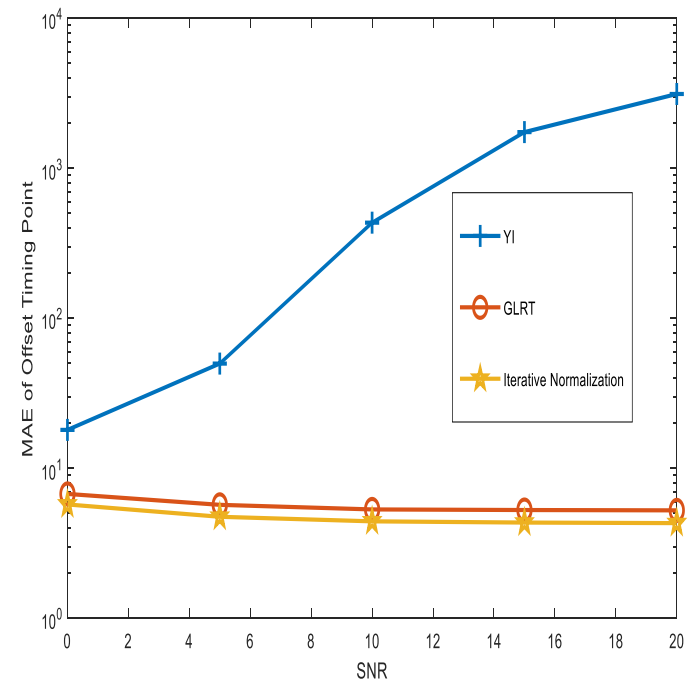

Figure 8. Simulation results for timing estimation measure in MAE for $\mathrm{SIR}=15 \mathrm{~dB}$.

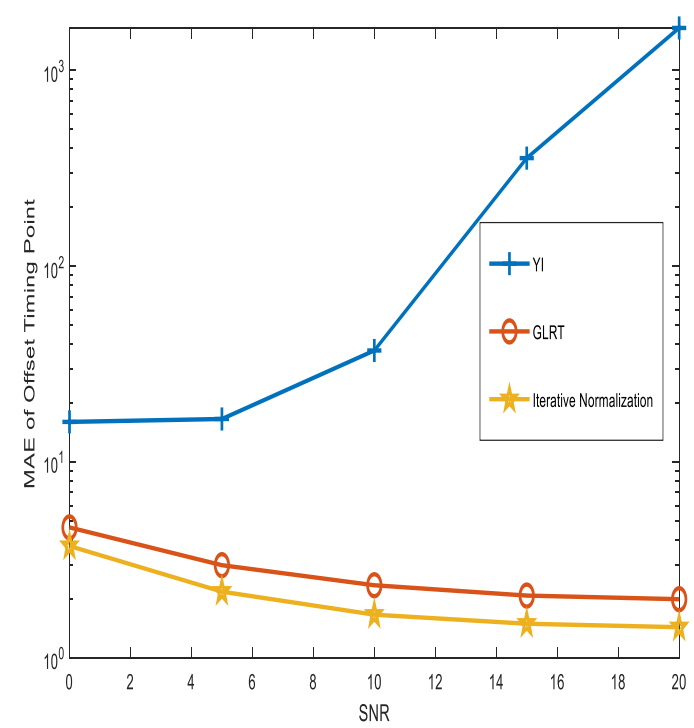

Figure 9. Simulation results for timing estimation measure in MAE for SIR $=20 \mathrm{~dB}$.

\section{MSE Evaluation}

Figures 10, 11, 12, 13, and 14 show the results of MAE simulation for $\mathrm{SIR}=0 \mathrm{~dB}, \mathrm{SIR}=5 \mathrm{~dB}, \mathrm{SIR}=10$ $\mathrm{dB}, \mathrm{SIR}=15 \mathrm{~dB}$, and SIR $=20 \mathrm{~dB}$. YI algorithm based on autocorrelation technique experiences a decrease in performance as SIR decreases. YI algorithm also decreases with increasing SNR, this is due to the higher NBI effect when SNR rises. In the GLRT Algorithm and Iterative Normalization Algorithm the performance increases when the SNR rises, this proves that both algorithms are more resilient than the YI algorithm against the influence of NBI. The performance of the Iterative Normalization algorithm is almost the same as the performance of the GLRT algorithm at SIR $\leq 10 \mathrm{~dB}$, whereas for $\mathrm{SIR} \geq 15 \mathrm{~dB}$ the performance of the iterative normalization algorithm is better than the GLRT algorithm. That is because normalization is done repeatedly, resulting in better performance. 


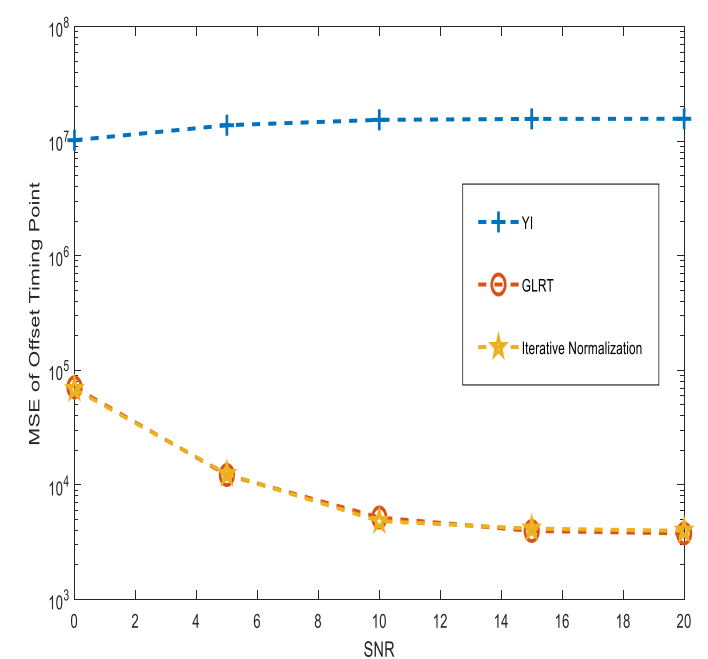

Figure 10. Simulation results for timing estimation measure in MSE for $\operatorname{SIR}=0 \mathrm{~dB}$.

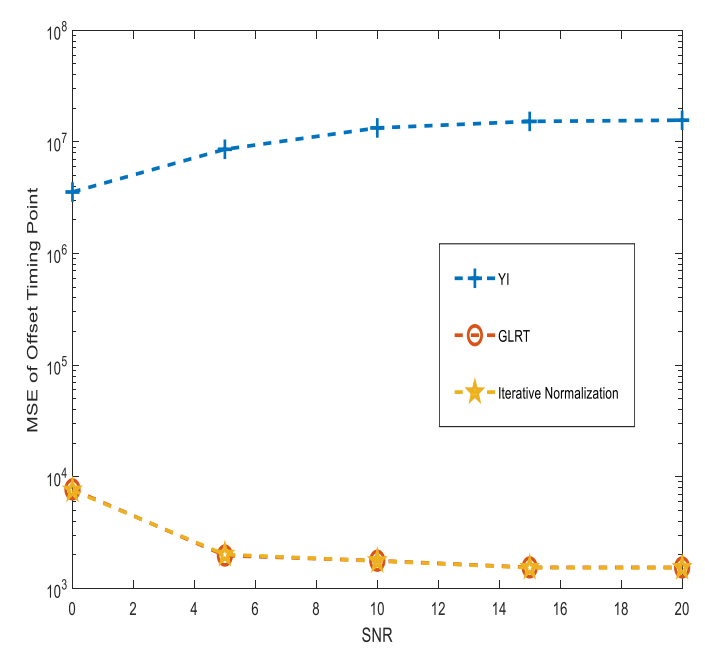

Figure 11. Simulation results for timing estimation measure in MSE for $\mathrm{SIR}=5 \mathrm{~dB}$.

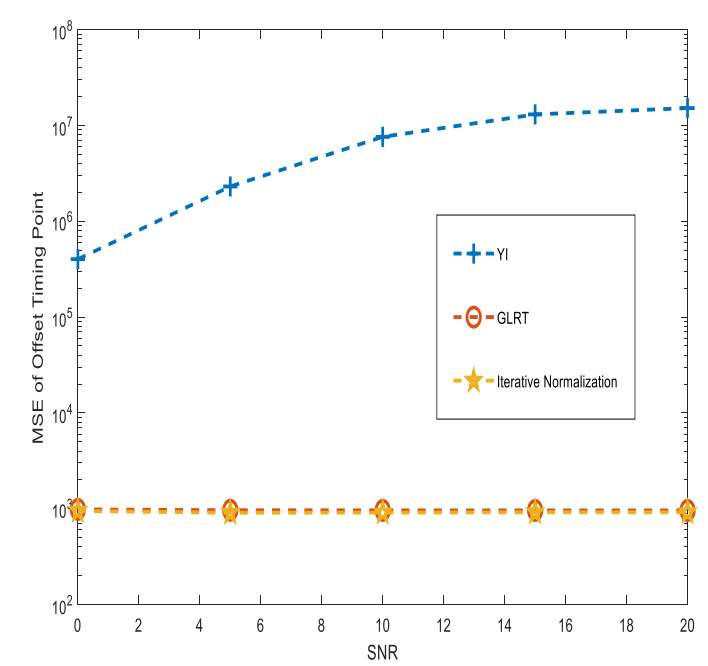

Figure 12. Simulation results for timing estimation measure in MSE for $\mathrm{SIR}=10 \mathrm{~dB}$.

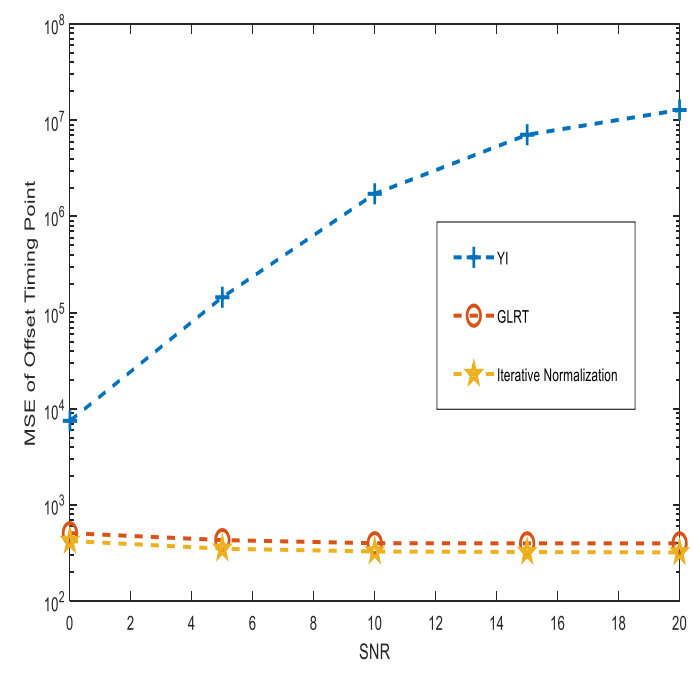

Figure 13. Simulation results for timing estimation measure in MSE for $\mathrm{SIR}=15 \mathrm{~dB}$.

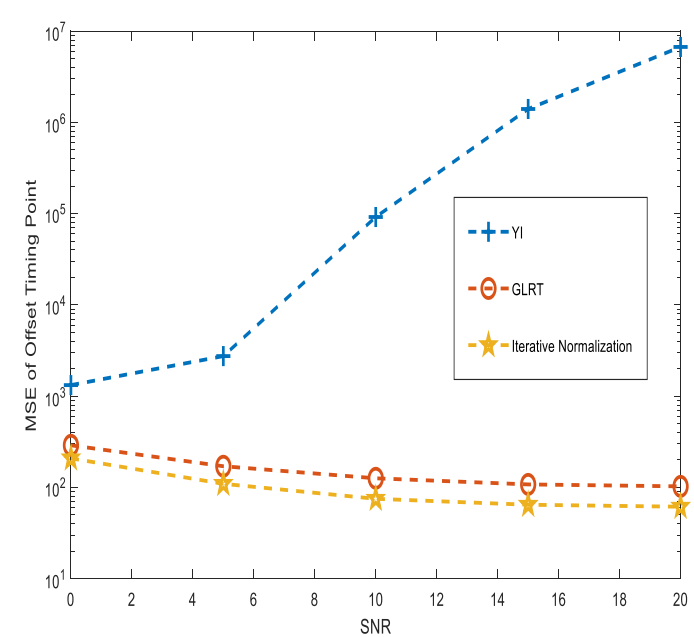

Figure 14. Simulation results for timing estimation measure in MSE for $\mathrm{SIR}=20 \mathrm{~dB}$.

\section{Complexity Analysis}

Table 3 shows the level of complexity of each algorithm. The Yi algorithm has the lowest complexity. The Iterative Normalization Algorithm is a little more complex than the GLRT algorithm. The overall performance of the Iterative Normalization algorithm is better than the YI and GLRT algorithms, although it must be paid with a slight increase in complexity.

TABLE 3

COMPLEXITY ANALYSIS OF THREE ALGORITHMS

\begin{tabular}{|l|c|c|}
\hline \multicolumn{1}{|c|}{ Algorithm } & \multicolumn{1}{|c|}{$\begin{array}{c}\text { Real additions/ } \\
\text { subtractions }\end{array}$} & $\begin{array}{c}\text { Real } \\
\text { multiplications } \\
\text { /divisions }\end{array}$ \\
\hline YI [7] & $N$ & $2 N+2$ \\
\hline GLRT [8] & $N+J-4$ & $2 N-1$ \\
\hline $\begin{array}{l}\text { Iterative } \\
\text { Normalization } \\
\text { [9] }\end{array}$ & $N+J+2 N_{\text {norm }}-6$ & $2 N-1$ \\
\hline
\end{tabular}




\section{CONCLUSION}

From the results of the time synchronization simulation performed for the NC-OFDM systems for Cognitive Radio for the three algorithms, it was found that the autocorrelation-based method has very poor performance, this is due to the presence of NBI. Therefore, this method is not suitable for NC-OFDM based Cognitive Radio systems. As an alternative, the GLRT method or iterative Normalization method can be used for the NC-OFDM Cognitive Radio system.

\section{ACKNOWLEDGMENT}

This paper was created in order to guide the first researcher (Tajul Miftahushudur) in writing scientific papers.

\section{REFERENCES}

[1] J. Ma, G. Y. Li, and B. H. Juang, "Signal processing in cognitive radio," in Proc. IEEE, vol. 97, no. 5, pp. 805-823, May 2009.

[2] H. A. Mahmoud, T. Yucek, and H. Arslan, "OFDM for cognitive radio: Merits and challenges," IEEE Wireless Commun., vol. 16, no. 2, pp. 6-15, Apr. 2009.
[3] T. M. Schmidl and D. C. Cox, "Robust frequency and timing synchronization for OFDM," IEEE Trans. Commun., vol. 45, no. 12, pp. 1613-1621, 1997.

[4] A. J. Coulson, "Narrowband interference in pilot symbol assisted OFDM systems," IEEE Trans. Wireless Commun., vol. 3, no. 6 pp. 2277-2287, Nov. 2004.

[5] M. Marey and H. Steendam, "Analysis of the narrowband interference effect on OFDM timing synchronization," IEEE Trans. Signal Process., vol. 55, no. 9, pp. 4558-4566, Sep. 2007.

[6] B. Park and H. Cheon, C. Kang, and D. Hong, "A novel timing estimation method for OFDM systems," IEEE Commun. Lett., vol. 7, no. 5, pp. 239-241, May 2003.

[7] G. Yi, L. Gang, and G. Jianhua, "A novel timing and frequency synchronization scheme for OFDM systems," IEEE Trans. Consumer Electron., vol. 54, no. 2, pp. 321-325, May 2008.

[8] Y.-H. Cho and D.-J. Park, "Timing estimation based on statistical change of symmetric correlator for OFDM system," IEEE Commun. Lett., vol. 17, No. 2, pp. 397-400, May. 2013.

[9] Suyoto, Iskandar, Sugihartono, and A. Kurniawan, "Improved timing estimation using iterative normalization technique for OFDM systems," Int. J. Elect. Comput. Eng., vol. 7, no. 2, pp. 905-912, Apr. 2017.

[10] Guideline for evaluation of radio transmission technologies for IMT-2000, Recommendation ITU-R M. 1225, 1997. 